\title{
Fetomaternal outcome in parturient women with and without epidural labour analgesia
}

\author{
Anupama M K \\ Assistant Professor, Dept. of Anesthesiology, P.K. Das Medical College, Vaniamkulam, Kerala, India \\ *Corresponding Author: Anupama M K \\ Email: anu2bdoc@gmail.com
}

Received: $5^{\text {th }}$ July, 2018

Accepted: $19^{\text {th }}$ November, 2018

\begin{abstract}
Introduction: Labour analgesia is being preferred by the parturient women these days due to increased awareness, comfortness and low threshold to perceive pain. The effects of epidural and parenteral analgesia on duration and mode of deliveries have been studied since last few decades.

Aim was to study the effects of epidural labour analgesia on the duration of labour, mode of delivery, fetal outcome and patient satisfaction and comfortness. This was a prospective, comparative and observational study.

Material and Methods: One hundred parturient women with full term single live gestation with vertex presentation with spontaneous onset of labour, meeting the inclusion criteria were divided into two groups.

Group C: Parturient women who opted out of labour analgesia were taken as controls.

Group E: Parturient women who opted for labour analgesia were taken as cases.

Statistical Analysis: Independent- Samples t test, Cross tabs and Repeated Measure ANOVA were used. SPSS for windows (version 17.0) was employed for data analysis.

$\mathrm{p}<0.05$ was considered as significant and $\mathrm{p}<0.01$ considered as highly significant.

Results: Demographic parameters like age, weight, height, BMI were comparable between the two groups E and group C (p >0.05)

The gestational age between the groups $\mathrm{C}$ and $\mathrm{E}$ were comparable as $\mathrm{p}>0.05$

No significant difference in Bishops score between Group E and Group C ( $p>0.05)$

There was no difference stastistically in terms of mode of delivery (Normal delivery, Instrumental delivery, Caesarean delivery) between the groups.

Instrumental delivery was seen more in group E. No change in normal or ceasarean deliveries. Shortening of active phase was seen in group $\mathrm{E}\left(\mathrm{P}<0.01\right.$ from $2^{\text {nd }}$ hour $)$. Prolonged second stage of labour was seen in group $\mathrm{E}(\mathrm{P}=0.001)$ which is statistically significant.

Decreased pain score in group E with $\mathrm{p}$ value of 0.006 which is highly significant statistically. There was improvement of APGAR at 1 minute in group $\mathrm{E}$ with significant $\mathrm{p}$ value. No significant difference between the groups for APGAR score at 5 minute.

No significant difference between the groups in terms of requirement of NICU admission.

Conclusion: Many safe techniques are available for parturient women to bypass the painful labour process and enjoy the birth of the baby. Labour analgesia is one of the most commonly used method in developed countries. Due to lack of awareness and false perception of ending up in caesserian sections, labour analgesia has not gained much popularity in India. Our study aims to study the incidence of casserian sections, instrumental deliveries and the effects of labour analgesia in newborns.

Epidural is a regional technique which is safe as to reduce the labour pain and to experience the joy of motherhood, without pain. The prs and cons of labour analgesia should be explained to al labour women and to be given an option of opting it to enjoy the process of painless labour.
\end{abstract}

Keywords: Epidural, Labour analgesia, Fetomaternal outcome, Instrumental deliveries, Parturient women caesarean section, Bishop's score, APGAR score, NICU.

\section{Introduction}

Every women in labour can be made to enjoy her labour if it is made pain free. Labor is defined as a series of events that take place in the genital organs in an effort to expel the viable products of conception out of the womb through the vagina into the outer world. ${ }^{1}$ Pain is a unpleasant stimulus which produces fear and anxiety. Pathophysiological responses occur in the body during pain. Metabolic acidosis as a result of increased metabolic rate in the second stage of labour is transferred to the foetus. ${ }^{2}$

Effective pain relief reduces plasma noradrenaline, ${ }^{3}$ prevents metabolic acidosis by reducing the rate of rise of lactate and pyruvate, ${ }^{4}$ prevents the rise during 1 st and 2 nd stage of labour of 11-hydroxy corticosteroid. ${ }^{5}$ It decreases maternal oxygen consumption by upto $14 \% .^{5}$

Analgesia in the form of regional techniques like epidural can relieve some degree of pain and the associated adverse pathophysiological changes. Epidural analgesia has been used using mixture of low dose local anaesthetics and opioids to provide pain relief in labour, but has the drawbacks of delayed onset and motor blockade. Low dose epidural analgesia has gained wide spread acceptance as an approach to labour analgesia.

\section{Aims and Objectives}

Aim of our study is to analyse the effectiveness of epidural labour analgesia and the risk of adverse maternal and fetal outcome.

\section{Primary Objective}

To study the effects of epidural analgesia and to study the incidence of instrumental assisted or cesarean delivery and duration of second stage of labour. 


\section{Secondary Objective}

To analyse the effect of epidural labour analgesia on the fetal outcome by APGAR score at 1 and 5 minutes, incidence of meconium stained amniotic fliud, meconium aspiration syndrome, NICU stay/ admission.

\section{Material and Methods}

A comparative prospective hospital based observational clinical study with patients coming to department of Obstetrics and Gynaecology, Apollo BGS Hospital, Mysore, Karnataka.

\section{Study Population}

Parturient women with full term single live gestation with vertex presentation with spontaneous onset of labour.

\section{Inclusion Criteria}

1. Age 18 to 40 years.

2. Single live fetus at term gestation (37 to 40 weeks) having vertex presentation.

3. Primigravidae/ Multigravidae having Spontaneous onset of labour with Bishops Score (BS>6) on per vaginal examination.

\section{Exclusion Criteria}

1. Patient refusal

2. Pregnancy with multifetal gestation, Ante partum haemorrhage, Pre-eclampsia, malpresentations, preterm labour.

3. Medical disorders like diabetes, hypertensive disorders of pregnancy, cardiac disease in pregnancy, renal, liver disorders, neurologic or neuromuscular disorders.

4. Altered blood coagulation profile.

5. Infection at the injection site

6. Allergy to local anesthetics

\section{Sample Size}

Sample Size of 60

Confidence Level: $95 \%$

Confidence Interval: $5 \%$

As calculated using the Kish Leslie Formula:

Sample size $=\frac{\mathrm{z}^{2 *}(\mathrm{p}) *(\mathrm{q})}{\mathrm{d}^{2}}$

$\mathrm{Z}=\mathrm{Z}$ value

$\mathrm{p}=$ percentage picking a choice (hospital prevalence of $4 \%$ ), expressed as decimal;

$\mathrm{c}=$ confidence interval, expressed as decimal.

Sample size $=[1.96 \times 1.96 \times 0.04 \times 0.96] \div[0.05 \times 0.05]$ $\mathrm{Z}=1.96$, that is the value of $\mathrm{Z}$ corresponding to $95 \%$ confidence interval

This gave a sample size of 60 divided into 2 groups. The above sample size is calculated using sample size calculator available at http://www.surveysystem.com/ sscalc.html)

\section{Methodology}

The study was conducted after Institutional Ethical Committee approval.

100 parturient women with full term single live gestation with vertex presentation with spontaneous onset of labour, meeting the inclusion criteria were divided into two groups.
Group C: Parturient women who opted out of labour analgesia were taken as controls.

Group E: Parturient women who opted for labour analgesia were taken as cases.

After securing epidural catheter, women received bolus of $10 \mathrm{ml}$ of $0.125 \%$ bupivacaine followed by infusion of $5 \mathrm{ml} /$ hour of $0.125 \%$ of bupivacaine with $2 \mathrm{mcg}$ of fentanyl in each $\mathrm{ml}$ as epidural infusion.

On admission detailed history and informed written consent was taken after explaining procedure to the patients. An 18-gauge intravenous cannula was inserted under local anaesthetic infiltration and an infusion of Ringer's lactate $100 \mathrm{ml} / \mathrm{hr}$ was started. The patients were connected to multiparameter monitor for recording Heart rate (HR), non invasive measurements of systolic blood pressure, diastolic blood pressure, Mean arterial pressure (MAP), continuous electrocardiogram (ECG) monitoring, oxygen saturation, continuous fetal cardiotocogram.

A detailed examination of the respiratory and cardiovascular system followed by per abdomen examination, fetal heart rate and obstetric examination done. If the patient satisfies the inclusion criteria, parturient consent was taken for epidural analgesia. After all investigations and preparation, the parturient is placed in lateral position. Under aseptic precautions, epidural space identified and catheter inserted using 18 G Tuohy needle into via loss of resistance technique. About $2-4 \mathrm{~cm}$ of catheter is left in the identified space and continuous infusion $0.125 \%$ of Bupivacaine and fentanyl $2 \mathrm{mcg} / \mathrm{ml}$ at rate of $5 \mathrm{ml} /$ hour via a syringe pump by qualified Anaesthesiologist in L2-L3 space, as often required for the duration of labour.

Maternal pulse, Blood pressure, pulse oximetry continuously monitored. Abdominal examination done to assess the intensity, duration and frequency of uterine contractions and descent of the fetal head in fifths using Crichton's formula.

Duration and progress of labour noted using partogram; fetal heart rate monitored continuously. Per vaginal examination done to note cervical dilatation, effacement, station of the head to know the descent, colour of the liquor. Depending on the vitals and progress of labour it was decided if patient will go for normal/instrumental/caesarean delivery.

\section{Data Collected}

1. Maternal heart rate, maternal Mean Arterial Pressure, continuous fetal monitoring using cardiotocography.

2. Uterine contractions, per vaginal examination to assess progress of labour

3. Duration of active phase of first stage of labour in hours, duration of second stage of labour in minutes,

4. Mode of delivery(normal, instrumental-forceps or ventouse or caesarean delivery)

5. Pain score using Visual Analogue Scale ${ }^{6}$

6. Modified Bishop's score ${ }^{7}$

7. APGAR Score $^{8}$ 
8. Cases of Meconium stained amniotic fluid (MSAF), Meconium aspiration syndrome (MAS)

9. Cases requiring NICU stay/admissions

10. Side effects of epidural like nausea, vomiting, pruritis, bradycardia, dryness of mouth, sedation, urinary retention will be noted.

\section{Results}

The demographic profile of both the groups showed no significant differences statistically with respect to age ( $\mathrm{p}=$ $0.850)$ weight $(\mathrm{p}=0.374)$, height $(\mathrm{p}=0.713)$ and $\mathrm{BMI}(\mathrm{p}=0$. 411).

There is no significant difference between the group E and group $\mathrm{C}$ in terms of gestational age $(\mathrm{p}>0.05)$.

The mean Bishops score of group E was 9.06 \pm 11.40 and group $\mathrm{C}$ was $7.54 \pm .50$ with $\mathrm{p}$ value $>0.05$, with no significant difference in Bishops score between Group $\mathrm{E}$ and Group C.

The mean of group E showing the mode of delivery was $1.44 \pm .70$ and mean of group $\mathrm{C}$ was $1.50 \pm .83$ respectively.

$\mathrm{p}$ value of group $\mathrm{E}$ and group $\mathrm{C}$ with respect to normal delivery was 0.6642 which was statistically not significant

$\mathrm{p}$ value of group $\mathrm{E}$ and group $\mathrm{C}$ in terms of instrumental delivery (forceps or ventouse) was 0.0384 which was statistically significant $(\mathrm{p}<0.05)$.

$\mathrm{p}$ value of group $\mathrm{E}$ and group $\mathrm{C}$ in terms of caesarean delivery was 0.1854 which is statistically not significant ( $\mathrm{p}>0.05)$.

The mean of group $\mathrm{E}$ regarding the duration of active phase of labour was $9.04 \pm 11.40$ and group $C$ was $7.52 \pm .50$ respectively.

There was statistically highly significant difference between groups regarding duration of active phase of labour as $p$ value $<0.01$ from $2^{\text {nd }}$ hour of duration. $p$ value was 0.3173 in the first hour. Hence no statistically significant difference but it was significant clinically. The statistical analysis shows there was shortening of active labour phase in group $\mathrm{E}$ compared to group $\mathrm{C}$.

The mean of group $\mathrm{E}$ and group $\mathrm{C}$ in terms of duration of second phase labour were $73.21 \pm 32.49$ and $53.52 \pm 21.09$ respectively.

There was statistically highly significant difference between the groups regarding the duration of second phase of labour in all variables as $\mathrm{p}$ value $<0.05$ throughout. The statistical analysis shows prolongation of second phase of labour in group $\mathrm{E}$ than group $\mathrm{C}$.

Mean pain score of group $\mathrm{E}$ and group $\mathrm{C}$ were $1.96 \pm .79$ and $2.72 \pm 1.46$ respectively with $p$ value: 0.006 which was highly significant showing that there was decreased pain score in group $\mathrm{E}$ than group $\mathrm{C}$.

$\mathrm{p}$ value between group $\mathrm{E}$ and group $\mathrm{C}$ for APGAR at 1 minute was 0.023 which is statistically significant. There was better APGAR score at 1 minute in group $E$ than group C.

$\mathrm{p}$ value between group $\mathrm{E}$ and group $\mathrm{C}$ for APGAR at 5 minutes was 0.156 which is clinically significant but statistically not significant. p-value between group $\mathrm{E}$ and group $\mathrm{C}$ requiring NICU admission was 0.697 which was statistically not significant.

There were no cases of Meconium stained amniotic fluid or Meconium aspiration syndrome.

Post-delivery maternal vitals were stable in both the groups.

\section{Salient Observations and findings of Our Study}

The demographic parameters like age, height, weight, BMI, Gestational age were comparable between both the groups.

Bishop's score, normal or ceasarean deliveries, incidence of instrumental (forceps or ventouse): no significant difference between the two groups.

Active phase of labour duration shortened with prolongation of second phase of labour.

Pain score decreased in group E (highly significant)

Improvement in APGAR at 1 minute in group E.

No change in APGAR at 5 minute between the two groups.

No significant difference in NICU admissions or stay.

No cases of Meconium stained amniotic fluid or Meconium aspiration syndrome.

Two cases in group E had nausea and vomiting which was treated symptomatically.

Four cases in group $\mathrm{C}$ had nausea which was treated symptomatically.

\section{Discussion}

Epidural analgesia provides good analgesia during first and second stage of labour than parenteral opioid. ${ }^{16}$

In our study, Epidural analgesia was given after cervical dilatation of $4 \mathrm{~cm}$ as per American college of obstetrician and gynecologists recommendations. ${ }^{22}$

In our study, the duration of $1^{\text {st }}$ stage of labour was shortened in the epidural group. This correlated with the studies done by Wong et $\mathrm{al}^{23}$ and Halpern et al. ${ }^{12}$

In our study, 2nd stage was prolonged in epidural group $\mathrm{E}$ as compared to control group $\mathrm{C}(\mathrm{p}<0.05)$. Similar results were seen in studies of Halpern et al, ${ }^{12}$ Barbara et al, ${ }^{16}$ Decca L, Daldoss C, Fratelli $\mathrm{N}$ et al, ${ }^{17}$ Halvadia SH, Halvadia BH, Joshi RM, Upadhyaya DP,${ }^{20}$ Dipti Agrawal and colleagues. ${ }^{22}$

In our study, statistically significant difference was found between epidural group $\mathrm{E}$ and control group $\mathrm{C}$ in the rates of instrumental vaginal (forceps or vacuum assisted) deliveries $(p<0.05)$ but no difference in caesarean and normal vaginal deliveries.

This correlates with findings of Philipsen T, Jensen $\mathrm{NH}$ ${ }^{11}$, Howell et al,${ }^{14}$ Sharma et al, ${ }^{15}$ Decca L, Daldoss C, Fratelli $\mathrm{N}$ et al, ${ }^{17}$ Hegazy, ${ }^{18}$ Khurshid et al, ${ }^{19}$ Halvadia SH, Halvadia $\mathrm{BH}$, Joshi RM, Upadhyaya $\mathrm{DP}^{20}$ where there was significant increase in instrumental vaginal (forceps or vacuum assisted) deliveries.

Several retrospective trials showed an association between the use of epidural and increased caesarean rate. ${ }^{24}$ But recent randomized studies do not show such increase. This correlates with studies of Chestnut et al. ${ }^{10}$ Philipsen T, 
Jensen NH, ${ }^{11}$ Zhang J, Klebanoff MA, Dersimonian $\mathrm{R},{ }^{13}$ Dipti Agrawal and colleagues. ${ }^{22}$

In our study 5 cases were taken for caeserean section, indications being cervical dystocia and non-progress of labour.

Our results demonstrated no significant difference in neonatal outcome (APGAR score at 1 and 5 minutes) between epidural and control groups. This correlates with previous studies like Blayaert et al, ${ }^{9}$ Chestnut et $a l,{ }^{10}$ Barbara et al, ${ }^{16}$ Halvadia SH, Halvadia BH, Joshi RM, Upadhyaya DP. ${ }^{20}$

Epidural analgesia when given with cervical dilatation of more than $4 \mathrm{~cm}$ provides better analgesic effect with minimal motor blockade of abdomino-pelvic muscles and encourages parturient to actively participate in labour helping the expulsion of fetus without pain.

\section{Conclusion}

Epidural labour analgesia provides a safe and good alternative for a painful labour.

All pregnant patients during her antenatal visits should be explained about the pros and cons of epidural labour analgesia given an option to opt for, if willing.

\section{Limitations of our Study}

Since the patient was asked for epidural analgesia, randomization could not be done.

Blinding was not possible.

\section{Recommendations}

Epidural labour analgesia should be offered to all patients to provide a painless labour and to experience the joy of childbirth without pain where the facilities exist.

\section{Conflict of Interest: None.}

\section{References}

1. Dutta DC. Text book of obstetrics. Chapter 12. $6^{\text {th }}$ edn. New Delhi: Hiralal Konar New Central Book Agency; 2004. pp11444.

2. Holdcroft A. Regional Anaesthetic Techniques. Chapter 15. In: Principles and practice of obstetric anaesthesia and analgesia. England: Blackwell Science Publishers; 2000. pp243-59.

3. Falconer AD, Powles AB. Plasma noradrenaline levels during labour - influence of elective lumbar epidural blockade. Anaesth 1982;37:16-8.

4. Marx GF, Greene NM. Maternal lactate, pyruvate and excess pyruvate production during labour and delivery. Am J Obstet Gynecol 1964;90:786-89.

5. Sanguol F, Fox GS, Houle GL. Effects of regional analgesia on maternal oxygen consumption during the first stage of labour. Am J Obstet Gynecol 1975; 121:1086-9.

6. Vas-visual analogue score. Available at http://www.jdentlasers.org/articles/ 2013/7/1/images/ J Dent Lasers_2013_7_1_27_118444_u2.jpg

7. Royal College of Obstetricians and Gynaecologists. RCOG Clinical Effectiveness Support Unit. Induction of labour. Evidence-based Clinical Guideline Number 9. London: RCOG Press; 2001.

8. Papile LA. The APGAR score in the $21^{\text {st }}$ century. $N$ Engl $J$ Med 2001;344:519-20.
9. Bleyaert A, Soetens M, Vaes L, Van Steenberg AL, Van der Donck A. Bupivacaine, 0.125 per cent, in obstetric epidural analgesia: experience in three thousand cases. Anaesth 1979;51(5):435-8.

10. Chestnut DH. Continuous infusion epidural analgesia during labor: A randomized double-blind comparison of $0.0625 \%$ bupivacaine $/ 0.0002 \%$ fentanyl vs $0.065 \%$ bupivacaine. Anaesth 1988;68:754-9.

11. Philipsen T, Jensen NH. Epidural block or parenteral pethidine as analgesic in labour: a randomized study concerning progress in labour and instrumental deliveries. Eur J Obstet Gynecol Reprod Biol 1989;30:27-33.

12. Halpern SH, Leighton BL Ohlsson A, Barrett JF, Rice A Effect of epidural vs parenteral opioid analgesia on the progress of labour: a meta-analysis. JAMA 1998;280:2105-10.

13. Zhang J, Klebanoff MA, Dersimonian R. Epidural analgesia in association with duration of labour and mode of delivery: a quantitative review. Am J Obstet Gynecol 1999;180:970-7.

14. Howell CJ, Kidd C, Roberts W, Upton P, Lucking L, Jones $\mathrm{PW}$, et al. A randomised controlled trial of epidural compared with non-epidural analgesia in labour. Br J Gynaecol 2001;108(1);27-3.

15. Sharma KS, Alexander MJ, Messick G, Bloom SL, McIntire $\mathrm{DD}$, Wiley J, et al. A randomized trial of epidural analgesia versus intravenous meperidine analgesia during labour in nulliparous women. Anesth 2002;96(3):546-51.

16. Leighton BL, Halpern SH. The effects of epidural analgesia on labour, maternal, and neonatal outcomes: A systematic review. Am J Obstet Gynecol 2002;186:S69-77.

17. Decca L, Daldoss C, Fratelli N, Lojacono A, Slompo M, Stegher C, et al. Labour course and delivery in epidural analgesia: a case-control study. J Matern Fetal Neonatal Med 2004;16(2):115-8.

18. Bakhamees H, Hegazy E. Does epidural increase the incidence of caesarean delivery or instrumental labour in Saudi populations? Middle East J Anesth 2007;19(3):693-704.

19. Halvadia SH, Halvadia HB, Joshi RM, Upadhyaya DP. A Study of fetomaternal outcome of epidural Analgesia during labor. Natl J Med Res 2013;3(2):184-6.

20. Agrawal D, Makhija B, Arora M, Haritwal A, Gurha P. The effect of epidural analgesia on labour, mode of delivery and neonatal outcome in nullipara of India, 2011-2014. J Clin Diagn Res 2014;8(10):OC03-OC06.

21. Goetzl LM. ACOG Practice Bulletin. Clinical Management Guidelines for Obstetrician-Gynecologist Number 36, July 2002. Obstetric Analgesia and Anesthesia. Obstet Gynecol 2002;100:177-91.

22. Wong CA, Scavone BM, Peaceman AM. The risk of caesarean delivery with neuraxial analgesia given early versus late in labour. N Engl J Med 2005;352:655-65.

23. Sharma SK, Leveno KJ. Regional analgesia and progress of labour. Clin Obstet Gynecol 2003;46:633-45.

How to cite this article: Anupama MK. Fetomaternal outcome in parturient women with and without epidural labour analgesia. Indian J Clin Anaesth 2019;6(2):222-3. 